\title{
REPORT ON A CASE OF SO-CALLED COLOBOMA OF THE MACULA
}

\author{
BY
}

Lt.-Col. A. M. DiCK, I.M.S. AND M. R. SAWhney, M.B.(Cantab.)

LAHORE

C. B., aged 43, a Hindu, attended the out-patient department of the Mayo Hospital, Lahore, on May 15, 1928, for defective vision. His distant vision was $2 / 60$ in each eye. Near vision for each eye was Jaeger 1 , at 4 inches. Contrary to what would ordinarily be expected in a person with the above vision there was no improvement with concave glasses, in fact they made the vision distinctly worse. After retinoscopy under homatropine his vision with plus $6 \mathrm{D}$. sphere and plus $2 \mathrm{D}$. cylinder, axis $180^{\circ}$, béfore each eye came up to 6/60 in each eye. There was no improvement in near vision.

On examination of the fundus it was found that it was quite normal except in the region of the mácula in each eye. In the left eye the fundus in the macular region showed an oval white patch with the long axis of the oval vertical. The difference between the two axes being very little, the patch approximated to a circle. The size of this patch was less than half the size of the disc. On careful examination it was seen that this patch did not include the fovea centralis, which could just be seen a little to the nasal-side of the upper limit of the patch. Retinal blood vessels stopped short near the margin of the patch, none coursing over it. There was no measurable ectasia of the patch. At the periphery of the patch and forming part of it were seen a number of small, round, white bodies which reflected light rather strongly and hence shone brightly under ophthalmoscopic examination. They had the appearance of crystals of cholesterine. Four of these bright white bodies on the nasal side and one in the upper part of the patch were the brightest. Occasionally during the examination, the whole of the patch seemed to shine like a phosphorescent body. Probably this was due to its reflecting light strongly at a certain angle. The rest of the patch looked white, stippled in places with very fine light grey points. On examination with a stronger light, such as that given by a Morton pattern Hamblin ophthalmoscope when light is reduced to a slit, there could be seen in the patch faintly but distinctly portions of two blood vessels, running horizontally from the periphery of the patch towards its centre for about one third of the width of the patch. One of these vessels came from the temporal and the other from the nasal side of the patch, neither of them quite reaching the periphery. These bits of vessels were probably the remains of the larger blood vessels of the choroid. 
There was no pigmentation either in the patch itself or surrounding it. Its margins were very sharply defined and surrounded by a normal fundus.

The appearances in the right fundus were almost identical with those in the left. The size of the patch was practically the same as in the left, only it was inclined to be more circular. Similar shining bodies were found at the periphery of the patch as on the left side. The fovea could not be seen on the right side, but from the position of the patch and the appearances of its surroundings it could be seen distinctly that the patch involved part of the macula
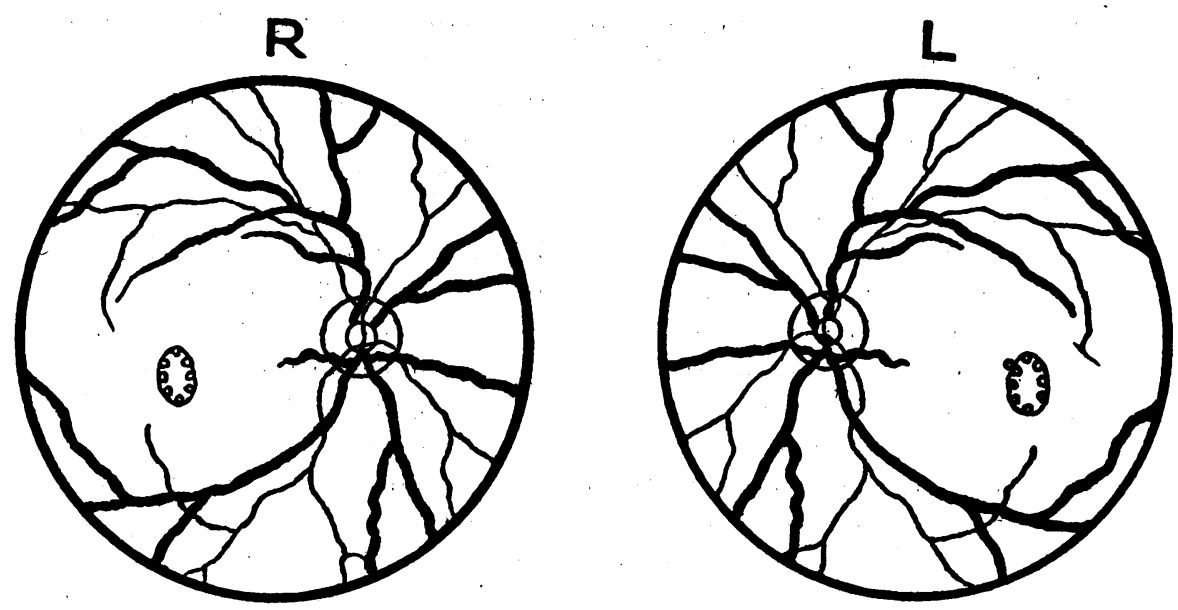

in the upper part. No blood vessels could be seen on this side. Beyond this there was no difference on the two sides and on a cursory examination the two fundi looked almost identical.

There was no contraction of the field of vision. Although the central vision was deficient, a scotoma could not be marked out on the perimeter, chiefly on account of lack of intelligence on the part of the patient, as he did not fix properly. When, however, a finger was held in front of his eye he said that he could see distinctly the two proximal phalanges of the finger but could not see the terminal phalanx distinctly, thus showing that he had a relative scotoma.

He had no other congenital defect in the eye.

He gave the history of having always been short sighted, that is ever since he could remember. He had never worn glasses nor worried about his sight as, although he was literate, he hardly ever did any reading as he was a farmer and tilled his own lands. He only came to the hospital as he had come to Lahore to see his sons. 
Two of his sons were examined. They had no defect beyond having a certain degree of refractive error. There was no history of syphilis either acquired or hereditary. It is of course impossible in these cases to be certain of the condition being congenital. But from the history and the appearances of the defect one could say that the condition was congenital or at least appeared soon after birth. There is little doubt that the case comes under the heading of so-called coloboma of the macula.

The chief points of interest in the case seem to be :

1. The size of the coloboma, it being less than half the size of the disc.

2. It was vertically oval and not horizontally oval as usual.

3. Its sharp definition.

4. The presence of round shining bodies which looked like cholesterine crystals, a degenerative product the result of an old haemorrhage or inflammation. This certainly goes in favour of the colobomata being pathological in origin.

According to Miss Ida Mann's classification this case comes under the heading of a non-pigmented coloboma.

\section{RETINAL DETACHMENT}

BY

RICHARD KERRY

MONTREAL

A REPORT of three cases of retinal detachment treated by the writer with iodized oil and resulting in restoration of vision was published in this journal some time ago, Vol. XII (May, 1928). Two cases treated recently by this method have not given the same happy result but as neither case was seen until the condition had been established for some months this is perhaps not surprising. There are, however, some points of interest connected with these cases which seem to be worth recording.

Of the first case, one of general detachment in the left eye of a young girl, for which no cause was established, there is little to be said, as treatment was discontinued after a few weeks. The superior temporal vein, however; which had been very dark and shrunken in appearance and with the blood column broken in many places, seemed when last seen, to be functioning well and was normal in appearance except for some displacement due to slight retinal oedema.

The second case, an over-stout, rheumatic woman of sixty odd, wearing - $8 \mathrm{D}$.sph. for the right eye and with the sight of the left 